\title{
WEIGHTED NORM INEQUALITIES FOR POTENTIAL OPERATORS
}

\author{
MARTIN SCHECHTER
}

ABSTRACT. We give sufficient conditions for inequalities of the form

$$
\left(\int\left(\int G(x-y) f(y) d \mu(y)\right)^{q} d \omega(x)\right)^{1 / q} \leq C\left(\int|f(y)|^{p} d \nu(y)\right)^{1 / p}
$$

to hold for measurable functions $f$. We determine the dependence of the constant $C$ on the measures $\mu, \nu, \omega$ and give some applications.

1. Introduction. Let $G(x)$ be a nonnegative function on $\mathbf{R}^{n}$, and let $\mu, \nu, \omega$ be Borel measures. If we define

$$
G d \mu(x)=\int G(x-y) d \mu(y)
$$

we shall be interested in inequalities of the form

$$
\|G f d \mu\|_{q, \omega} \leq C\|f\|_{p, \nu}
$$

where

$$
\|f\|_{p, \nu}=\left(\int|f(y)|^{p} d \nu(y)\right)^{1 / p}
$$

Such inequalities arise in many areas of mathematics and physics, and we shall be giving some applications.

In addition to the assumption that $G(x)$ be nonnegative, we shall also assume that there is a constant $C_{0}$ such that

$$
G(y) \leq C_{0} G(x) \quad \text { when }|x| \leq 2|y|
$$

This condition is satisfied in most cases of potential functions. For instance, the Riesz potential is given by

$$
I_{s}(x)=\Gamma\left(\frac{1}{2}(n-s)\right) 2^{-s} \pi^{-n / 2} \Gamma\left(\frac{1}{2} s\right)^{-1}|x|^{s-n}
$$

and the Bessel potential is given by

$$
G_{s}(x)=(4 \pi)^{-n / 2} \Gamma\left(\frac{1}{2} s\right)^{-1} \int_{0}^{\infty} e^{-t-|x|^{2} / 4 t} t^{[(s-n)-1] / 2} d t .
$$

Both of these functions are radial and decreasing in $|x|$. Hence they satisfy (1.3) with $C_{0}=1$.

Received by the editors June 20, 1986.

1980 Mathematics Subject Classification (1985 Revision). Primary 26D15; Secondary 42B25, $26 \mathrm{D} 10$.

Key words and phrases. Weighted norm inequalities, potential operators, maximal operators. 
For any function of sets $\sigma(Q)$, we define

$$
M_{\sigma, \delta} d \mu(x)=\sup _{\substack{x \in Q \\|Q| \leq \delta^{n}}} \sigma(Q)^{-1} \mu(Q)
$$

where the supremum is taken over all cubes $Q$ with sides parallel to the coordinate axes, containing the point $x$ and having volume $|Q| \leq \delta^{n}$. Here

$$
\mu(E)=\int_{E} d \mu(y) .
$$

We also take

$$
M_{\sigma} d \mu(x)=M_{\sigma, \infty} d \mu(x)=\sup _{x \in Q} \sigma(Q)^{-1} \mu(Q) .
$$

We consider finite cubes $Q$ with sides parallel to the coordinate axes. For a cube $Q$ we let $l(Q)$ denote the length of its side and we put $\gamma(Q)=4 \sqrt{n} l(Q)$. For $\alpha>0$ we let $\alpha Q$ denote the cube with side length $\alpha l(Q)$ and having the same center as $Q$. For a measure $\mu$ we let $\mu_{Q}$ denote the restriction of $\mu$ to $Q$.

Our first result is

THEOREM 1.1. Assume that $a, b, p, q, t>1, t<p \leq a$ and that

$$
1 / a+1 / b=1 / q \text {. }
$$

Assume also that $\mu$ is absolutely continuous with respect to $\nu$ and that

$$
\left\|G d \mu_{Q}\right\|_{q, \omega}<\infty
$$

for all $G$. Let

$$
\sigma([1+8 \sqrt{n}] Q)=\omega(Q) / \sup _{y} \int_{|x-y|<\gamma(Q) ; x \in Q} G(x-y) d \omega(x) ;
$$

and let $\rho, \tau$ be functions of sets such that

$$
\sigma(Q) \geq \rho(Q)^{1 / t} \tau(Q)^{1 / t^{\prime}}
$$

for any cube $Q$, where $t^{\prime}=t /(t-1)$. Then there is a constant $C$ depending only on $p, q$, and $n$ such that

$$
\begin{aligned}
\|G f d \mu\|_{q, \omega} \leq & C C_{0}^{q}\|f\|_{p, \nu}\left\|M_{\tau}(d \mu / d \nu)^{t^{\prime}} d \nu\right\|_{b / t^{\prime}, \omega}^{1 / t^{\prime}} \\
& \times \sup _{Q} \nu(Q)^{-1 / p}\left\|M_{\rho} \chi_{Q} d \nu\right\|_{a / t, \omega, Q}^{1 / t} .
\end{aligned}
$$

Variations of this theorem are given by

THEOREM 1.2. Under the same hypotheses

$$
\begin{aligned}
\|G f d \mu\|_{q, \omega} \leq & q^{-1} 2^{q+1}(q+1)^{1+1 / q} C_{0}^{q}\|f\|_{p, \nu} \\
& \times\left\|M_{\sigma^{p^{\prime}}}(d \mu / d \nu)^{p^{\prime}} d \nu\right\|_{q / p^{\prime}, \omega}^{1 / p^{\prime}}
\end{aligned}
$$

THEOREM 1.3. Under the same hypotheses, if $p \leq q$, then

$$
\|G f d \mu\|_{q, \omega} \leq C C_{0}^{q}\|f\|_{p, \nu} \sup _{Q}\|d \mu / d \nu\|_{p^{\prime}, \omega, Q}^{1-p^{\prime}}\left\|M_{\sigma \chi_{Q}}(d \mu / d \nu)^{p^{\prime}} d \nu\right\|_{q, \omega, Q}
$$


THEOREM 1.4. Under the same hypotheses, if $p \leq q$, then

$$
\begin{aligned}
\|G f d \mu\|_{q, \omega} \leq & C C_{0}^{q}\|f\|_{p, \nu} \sup _{Q}\left\|M_{\tau}(d \mu / d \nu)^{t^{\prime}} d \nu\right\|_{b / t^{\prime}, \omega, Q}^{1 / t^{\prime}} \\
& \times \sup _{Q}\left\|M_{\rho} \chi_{Q} d \nu\right\|_{a / t, \omega, Q}^{1 / t} \nu(Q)^{-1 / p} .
\end{aligned}
$$

The proofs of these theorems will be given in $\S 2$. The first step is to prove

$$
\|G f d \mu\|_{q, \omega} \leq C_{q}\left\|M_{\sigma} f d \mu\right\|_{q, \omega}
$$

where $C_{q}=q^{-1} 2^{q+1}(q+1)^{1+1 / q} C_{0}^{q}$ (Corollary 2.4). This estimate is obtained by a method of Muckenhoupt and Wheeden [3]. Then we find estimates for the righthand side (Theorems 2.7-2.10). Here we use, among other things, results of Sawyer [1].

An interesting consequence of these theorems is

COROLlary 1.5. Assume that (1.7) holds and that $\sigma$ is given by (1.8). If $1 \leq t<p \leq q$ and

$$
\tau(Q)=\int_{Q}(d \mu / d \nu)^{t^{\prime}} d \nu
$$

then

$$
\|G f d \mu\|_{q, \omega} \leq C \sup _{Q} \nu(Q)^{-1 / p}\left\|M_{\sigma^{t} \tau^{1-t}} \chi_{Q} d \nu\right\|_{q / t, \omega, Q}^{1 / t}\|f\|_{p, \nu} .
$$

Next we show how we can weaken (1.7). We let

$$
\widetilde{G}_{r} d \mu(x)=\int_{|x-y|>r} G(x-y) d \mu(y)
$$

and assume

$$
\left\|\widetilde{G}_{r} d \mu_{Q}\right\|_{q, \omega}<\infty \text { for some } r \text { and all } Q \text {. }
$$

We have

THEOREM 1.6. If (1.7) is replaced by (1.16), then Theorems 1.1-1.4 and Corollary 1.5 hold with $C_{q}$ replaced by $q^{-q} 2^{q+1}(q+1)^{q+1} C_{0}^{q}$.

Special cases of inequality (1.2) have been considered by several authors (cf. [1-25] and the references quoted in them). It does not appear that anyone has hitherto studied (1.2) in its full generality. An important aspect of our approach is that there are no preconditions placed on the measures $\mu, \nu, \omega$. Inequality (1.2) holds provided (1.16) holds and the constants in any of the inequalities (1.1), (1.12), (1.13), or (1.15) are finite.

As an application we consider the weighted Sobolev space $H^{s, p}\left(\mathbf{R}^{n}, d \nu\right)$ with norm given by

$$
\|u\|_{s, p, \nu}=\left\|\bar{F}\left(1+|\xi|^{2}\right)^{s / 2} F u\right\|_{p, \nu}
$$

where $F$ denotes the Fourier transform

$$
F u(\xi)=(2 \pi)^{-n / 2} \int_{\mathbf{R}^{n}} e^{-i \xi x} u(x) d x
$$

and $\bar{F}$ denotes its inverse. We shall be concerned with the case $s>0$. As an application of Corollary 1.5 we have the following theorem. 
THEOREM 1.7. Assume that $1 \leq t<p \leq q$ and that there are constants $\alpha<q$, $R$ such that

$$
\int_{|x|>R} e^{-\alpha|x|} d \omega(x)<\infty
$$

Let $\sigma$ be given by

$$
\sigma([1+8 \sqrt{n}] Q)=\omega(Q) / \sup _{y} \int_{|x-y|<\gamma(Q) ; x \in Q} G_{s}(x-y) d \omega(x)
$$

and let $\tau$ be given by

$$
\tau(Q)=\int_{Q}(d x / d \nu)^{t^{\prime}} d \nu .
$$

If the Lebesgue measure is absolutely continuous with respect to $\nu$, then

$$
\|u\|_{q, \omega} \leq C \sup _{Q} \nu(Q)^{-1 / p}\left\|M_{\sigma^{t} \tau^{1-t} \chi_{Q}} d \nu\right\|_{q / t, \omega, Q}^{1 / t}\|u\|_{s, p, \nu} .
$$

We can also obtain criteria for (1.2) to hold even when $q<p$ by using either Theorem 1.1 or Theorem 1.2. Moreover, we can have our inequalities depend on the operators $M_{\rho, \delta}$ with $\delta<\infty$ and the supremum taken over cubes $Q$ satisfying $|Q| \leq \delta^{n}$ if we assume

$$
\left(\int\left(\int_{|x-y|>r} G(x-y)^{q} d \omega(x)\right)^{p^{\prime} / q} \frac{d \mu^{p^{\prime}}}{d \nu} d \nu\right)^{1 / p^{\prime}}<\infty .
$$

This can be seen from the theorems of $\S 2$. The formulation of such theorems is straightforward and is left to the reader.

Proof of the theorems of this section will be found in $\S 2$.

After this paper was submitted for publication, Eric Sawyer gave the author a copy of his paper [26] in which he finds two conditions which together are necessary and sufficient for (1.2) to hold in the case $p \leq q, \mu=\nu$, and $G(x)$ is a positive semicontinuous radial function decreasing in $|x|$. In the present paper we do not necessarily make these restrictions. However, our conditions are only sufficient. It would be of interest to find the relationship between the results in the cases of overlap.

2. An inequality. In this section we shall prove a basic estimate that will be used in establishing the theorems of $\S 1$. First we have

THEOREM 2.1. Let $0<q<\infty$, and let $\mu, \omega$ be locally finite Borel measures on $\mathbf{R}^{n}$. Assume that the function $G(x)$ on $\mathbf{R}^{n}$ satisfies

$$
G(x) \geq 0
$$

and

$$
|x| \leq 2|y| \text { implies } G(y) \leq C_{0} G(x) .
$$

Then for every $\varepsilon>0$ and $\delta>0$

$$
\left\|G_{r} d \mu\right\|_{q, \omega}^{q} \leq \varepsilon^{q}\left\|G_{2 r} d \mu\right\|_{q, \omega}^{q}+\left(2^{q+1} C_{0}^{q} / \varepsilon^{q}\right)^{q}\left\|M_{\sigma, \delta} d \mu\right\|_{q, \omega}^{q}
$$


where

$$
\begin{gathered}
G_{r} d \mu(x)=\int_{|x-y|<r} G(x-y) d \mu(y), \\
M_{\sigma, \delta} d \mu(x)=\sup _{\substack{x \in Q \\
|Q| \leq \delta^{n}}} \sigma(Q)^{-1} \mu(Q), \\
\sigma([1+8 \sqrt{n}] Q)=\omega(Q) / \sup _{y} \int_{|x-y|<4 \sqrt{n} l(Q) ; x \in Q} G(x-y) d \omega(x),
\end{gathered}
$$

and

$$
r \leq 2 \sqrt{n} \delta /(1+8 \sqrt{n})
$$

PROOF. Let

$$
S_{\lambda}=\left\{x \in \mathbf{R}^{n} \mid G_{2 r} d \mu(x)>\lambda\right\}
$$

for any $\lambda$. If $S_{\lambda} \neq \mathbf{R}^{n}$, then

$$
S_{\lambda}=\bigcup_{j=1} Q_{j},
$$

where the cubes $Q_{j}$ have sides parallel to the coordinate axes, have disjoint interiors, and satisfy

$$
d\left(Q_{j}, S_{\lambda}^{c}\right) \leq 3 \sqrt{n} l\left(Q_{j}\right)
$$

where $M^{c}$ denotes the complement of $M$ in $\mathbf{R}^{n}$ (cf. e.g., [17, p. 10]). By subdividing $Q_{j}$ if necessary, we may require that

$$
\rho_{j} \equiv 4 \sqrt{n} l\left(Q_{j}\right) \leq 2 r
$$

If (2.10) is achieved by subdivision, we lose (2.9). But in this case we can also require

$$
r \leq \rho_{j}
$$

Thus we can make each $Q_{j}$ satisfy (2.10). If it does not satisfy (2.11), it will satisfy (2.9).

Let $b, d$ be positive numbers to be determined later. Define

$$
E_{j}=\left\{x \in Q_{j} \mid G_{r} d \mu(x)>\lambda b, M_{\sigma, \delta} d \mu(x) \leq \lambda d\right\}
$$

for each $j$. Let $Q$ be one of the cubes $Q_{j}$, and let $E \subset Q$ be the set given by (2.12). Assume first that $Q$ satisfies (2.10) and (2.11). Then we have

$$
\begin{aligned}
\lambda b \omega(E) & \leq \int_{Q} G_{r} d \mu(x) d \omega(x)=\int_{Q} \int_{|x-y|<r} G(x-y) d \mu(y) d \omega(x) \\
& =\int\left[\int_{|x-y|<r ; x \in Q} G(x-y) d \omega(x)\right] d \mu(y) \\
& \leq \sup _{y} \int_{|x-y|<\rho ; x \in Q} G(x-y) d \omega(x) \int_{Q+2 \rho} d \mu(y) \\
& \leq \omega(Q) M_{\sigma, l(Q)+2 \rho} d \mu(x), \quad x \in Q,
\end{aligned}
$$


by (2.6), where $\rho=\rho_{j}$. Assume that $E \neq \varnothing$. Since $l(Q)+2 \rho \leq \delta$ by $(2.7)$ and (2.10), there is an $x \in Q$ such that

$$
M_{\sigma, l(Q)+2 \rho} d \mu(x) \leq \lambda d .
$$

Thus

$$
\omega(E) \leq(2 d / b) \omega(Q)
$$

(the reason for the 2 will be given later).

Next assume that $Q$ satisfies (2.9) and (2.10) but not (2.11). Thus $\rho<r$, and there is a point $x_{0}$ and in $S_{\lambda}$ within a distance of $3 \sqrt{n} l(Q)$ of $Q$. Thus

$$
G_{2 r} d \mu\left(x_{0}\right) \leq \lambda \text {. }
$$

If $x \in Q$, then $\left|x-x_{0}\right|<\rho$. Hence if $|y-x|>\rho$, we have

$$
\left|y-x_{0}\right| \leq|y-x|+\left|x-x_{0}\right| \leq 2|y-x| \text {. }
$$

Thus if $x \in Q$, then

$$
\begin{aligned}
G_{r} d \mu(x) & =\int_{|x-y|<\rho}+\int_{\rho<|x-y|<r} G(x-y) d \mu(y) \\
& \leq G_{\rho} d \mu(x)+C_{0} \int_{\left|x_{0}-y\right|<2 r} G\left(x_{0}-y\right) d \mu(y) \\
& =G_{\rho} d \mu(x)+C_{0} G_{2 r} d \mu\left(x_{0}\right) \\
& \leq G_{\rho} d \mu(x)+C_{0} \lambda
\end{aligned}
$$

by (2.2) and (2.14). We now take $b=2 C_{0}$. Then

$$
E \subset\left\{x \in Q \mid G_{\rho} d \mu(x)>\frac{1}{2} b \lambda, M_{\sigma, \delta} d \mu(x) \leq d \lambda\right\} .
$$

Consequently,

$$
\begin{aligned}
\frac{1}{2} \lambda b \omega(E) & \leq \int_{Q} G_{\rho} d \mu(x) d \omega(x)=\int_{Q} \int_{|x-y|<\rho} G(x-y) d \mu(y) d \omega(x) \\
& =\int\left(\int_{|x-y|<\rho ; x \in Q} G(x-y) d \omega(x)\right) d \mu(y) \\
& \leq \omega(Q) M_{\sigma, l(Q)+2 \rho} d \mu(x), \quad x \in Q, \\
& \leq \omega(Q) \lambda d,
\end{aligned}
$$

by (2.6) and (2.7). Thus (2.13) holds in this case as well (here we need the factor of 2). If we now add (2.13) over all the cubes, we obtain

$$
\omega\left(\left\{G_{r} d \mu(x)>\lambda b, M_{\sigma, \delta} d \mu(x) \leq \lambda d\right\}\right) \leq(2 d / b) \omega\left(S_{\lambda}\right) .
$$

Thus

$$
\omega\left(\left\{G_{r} d \mu(x)>\lambda b\right\}\right) \leq(2 d / b) \omega\left(S_{\lambda}\right)+\omega\left(\left\{M_{\sigma, \delta} d \mu(x)>\lambda d\right\}\right)
$$

and consequently

$$
\begin{aligned}
\int_{0}^{N} & \omega\left(\left\{G_{r} d \mu(x)>\lambda b\right\}\right) d \lambda^{q} \\
& \leq \frac{2 d}{b} \int_{0}^{N} \omega\left(S_{\lambda}\right) d \lambda^{q}+\int_{0}^{N} \omega\left(\left\{M_{\sigma, \delta} d \mu(x)>\lambda d\right\}\right) d \lambda^{q}
\end{aligned}
$$


This gives

$$
\begin{aligned}
& \int_{0}^{N b} \omega\left(\left\{G_{r} d \mu(x)>\gamma\right\}\right) d \gamma^{q} \\
& \quad \leq 2 d b^{q-1} \int_{0}^{N} \omega\left(S_{\lambda}\right) d \lambda^{q}+\frac{b}{d^{q}} \int_{0}^{N d} \omega\left(\left\{M_{\sigma, \delta} d \mu(x)>\gamma\right\}\right) d \gamma^{q} .
\end{aligned}
$$

Letting $N \rightarrow \infty$, we obtain

$$
\left\|G_{r} d \mu\right\|_{q, \omega}^{q} \leq 2 d b^{q-1}\left\|G_{2 r} d \mu\right\|_{q, \omega}^{q}+(b / d)^{q}\left\|M_{\sigma, \delta} d \mu\right\|_{q, \omega}^{q} .
$$

If we now take $\varepsilon^{q}=2 d b^{q-1}, b=2 C_{0}$, we obtain (2.3).

COROLLARY 2.2. If $1 \leq q<\infty$ and

$$
\|G d \mu\|_{q, \omega}<\infty
$$

then for $0<\varepsilon<1$

$$
\|G d \mu\|_{q, \omega} \leq \frac{2^{q+1} C_{0}^{q}}{\left(1-\varepsilon^{q}\right)^{1 / q} \varepsilon^{q}}\left\|M_{\sigma} d \mu\right\|_{q, \omega}
$$

where

$$
M_{\sigma} d \mu(x)=M_{\sigma, \infty} d \mu(x)=\sup _{x \in Q} \sigma(Q)^{-1} \mu(Q) .
$$

ProOF. We note that

$$
G_{r} d \mu \leq G d \mu, \quad M_{\sigma, \delta} d \mu \leq M_{\sigma} d \mu
$$

Thus (2.3) implies

$$
\left\|G_{r} d \mu\right\|_{q, \omega}^{q} \leq \varepsilon^{q}\|G d \mu\|_{q, \omega}^{q}+\left(2^{q+1} C_{0}^{q} / \varepsilon^{q}\right)^{q}\left\|M_{\sigma} d \mu\right\|_{q, \omega}^{q} .
$$

Since $G_{r} d \mu \nearrow G d \mu$ the result follows.

COROLlaRY 2.3. If $1 \leq q<\infty$ and

$$
\left\|G d \mu_{Q}\right\|_{q, \omega}<\infty
$$

for each finite cube $Q$, then

$$
\|G d \mu\|_{q, \omega} \leq q^{-1} 2^{q+1} C_{0}^{q}(q+1)^{1+1 / q}\left\|M_{\sigma} d \mu\right\|_{q, \omega} .
$$

PROOF. Let $\left\{Q_{k}\right\}$ be a sequence of cubes such that $\chi_{Q_{k}} \rightarrow 1$, and let $d \mu_{k}=$ $\chi_{Q_{k}} d \mu$. Then $\left\|G d \mu_{k}\right\|_{q, \omega}<\infty$ for each $k$. Thus by Corollary 2.2, inequality (2.17) holds for $\mu_{k}$. Since $M_{\sigma} d \mu_{k} \leq M_{\sigma} d \mu$ and $G d \mu_{k} \nearrow G d \mu$, inequality (2.7) holds for $\mu$. The constant in (2.20) is obtained by minimizing the constant in (2.17).

COROLlaRY 2.4. If $1 \leq q<\infty$ and (2.19) holds, then

$$
\|G f d \mu\|_{q, \omega} \leq C_{q}\left\|M_{\sigma} f d \mu\right\|_{q, \omega}
$$

for all measurable functions $f \geq 0$, where $C_{q}$ is the constant in (2.20).

PROOF. For each $f$, let $\left\{f_{k}\right\}$ be a sequence of simple functions with compact supports such that $0 \leq f_{k} \nearrow f$. By $(2.19)$

$$
\left\|G f_{k} d \mu_{Q}\right\|_{q, \omega}<\infty
$$

for each $k$. Thus by Corollary 2.3

$$
\left\|G f_{k} d \mu\right\|_{q, \omega} \leq C_{q}\left\|M_{\sigma} f_{k} d \mu\right\|_{q, \omega} .
$$

Since $M_{\sigma} f_{k} d \mu \leq M_{\sigma} f d \mu$ and $G f_{k} d \mu \nearrow G f d \mu$, the result follows. 
PROPOSITION 2.5. Suppose $\mu$ is absolutely continuous with respect to $\nu, 1<$ $r<\infty$, and $\rho, \sigma, \tau$ are functions of sets such that

$$
\sigma(Q) \geq \rho(Q)^{1 / r} \tau(Q)^{1 / r^{\prime}}
$$

Then

$$
M_{\sigma, \delta} f d \mu(x) \leq\left[M_{\rho, \delta} f^{r} d \nu(x)\right]^{1 / r}\left[M_{\tau, \delta} \frac{d \mu^{r^{\prime}}}{d \nu} d \nu(x)\right]^{1 / r^{\prime}}
$$

PROOF. We note that for any cube $Q$

$$
\sigma(Q)^{-1} \int_{Q} f d \mu \leq\left[\rho(Q)^{-1} \int_{Q} f^{r} d \nu\right]^{1 / r}\left[\tau(Q)^{-1} \int_{Q} \frac{d \mu^{r^{\prime}}}{d \nu} d \nu\right]^{1 / r^{\prime}}
$$

Apply the definition.

If we use the notation

$$
\|f\|_{p, \lambda, Q}=\left(\int_{Q}|f(x)|^{p} d \lambda(x)\right)^{1 / p}
$$

we have

COROLlary 2.6. Under the hypotheses of Proposition 2.5

$$
\left\|M_{\sigma, \delta} f d \mu\right\|_{q, \omega, Q} \leq\left\|M_{\rho, \delta} f^{r} d \nu\right\|_{a / r, \omega, Q}^{1 / r}\left\|M_{\tau, \delta} \frac{d \mu^{r^{\prime}}}{d \nu} d \nu\right\|_{b / t^{\prime}, \omega, Q}^{1 / r^{\prime}}
$$

where $a, b \geq q$ satisfy

$$
1 / a+1 / b=1 / q
$$

The following was essentially proved by Sawyer $[\mathbf{1}]$.

THEOREM 2.7. Assume that $1<p \leq q \leq \infty, p<\infty$. Then

$$
\left\|M_{\sigma, \delta} f d \mu\right\|_{q, \omega} \leq C\|f\|_{p, \nu}
$$

holds for all $f$ if and only if $\mu$ is absolutely continuous with respect to $\nu$ and

$$
\left\|M_{\sigma, \delta} \chi_{Q} \frac{d \mu^{p^{\prime}}}{d \nu} d \nu\right\|_{q, \omega, Q} \leq C^{\prime}\left\|\frac{d \mu}{d \nu}\right\|_{p^{\prime}, \nu, Q}^{p^{\prime} / p}
$$

hold for all cubes $Q$ with $|Q| \leq \delta^{n}$. 
A variation of this is

THEOREM 2.8. Assume that $1<p, q<\infty$ (we can allow $q<p$ ). Let $r, a, b$ be numbers such that (2.26) holds and

$$
1<r<p \leq a .
$$

Let $\rho, \tau$ be the set function satisfying (2.22). Then there is a constant $C$ depending only on $p, q$, and $n$ such that

$$
\begin{aligned}
\left\|M_{\sigma, \delta} f d \mu\right\|_{q, \omega} \leq & C\left\|M_{\tau, \delta}(d \mu / d \nu)^{r^{\prime}} d \nu\right\|_{b / r^{\prime}, \omega}^{1 / r^{\prime}}\|f\|_{p, \nu} \\
& \times \sup _{|Q| \leq \delta^{n}} \nu(Q)^{-1 / p}\left\|M_{\rho, \delta} \chi_{Q} d \nu\right\|_{a / r, \omega, Q}^{1 / r} .
\end{aligned}
$$

ProOF. By Theorem 2.7

$$
\left\|M_{\rho, \delta} f^{r} d \nu\right\|_{a / r, \omega}^{1 / r} \leq C\left\|f^{r}\right\|_{p / r, \nu}^{1 / r} \sup _{|Q| \leq \delta^{n}} \nu(Q)^{-1 / p}\left\|f_{\rho, \delta} \chi_{Q} d \nu\right\|_{a / r, \omega, Q}^{1 / r}
$$

If we now make use of Corollary 2.6, we obtain (2.30).

THEOREM 2.9. Under the same hypotheses

$$
\left\|M_{\sigma, \delta} f d \mu\right\|_{q, \omega} \leq\left\|M_{\sigma^{p^{\prime}, \delta}}(d \mu / d \nu)^{p^{\prime}} d \nu\right\|_{q / p^{\prime}, \omega}^{1 / p^{\prime}}\|f\|_{p, \nu} .
$$

ProOF. Take $r=p, \rho=1, \tau=\sigma^{p^{\prime}}$ in Proposition 2.5. Then we have

$$
M_{\sigma, \delta} f d \mu(x) \leq\|f\|_{p, \nu}\left[M_{\sigma^{p^{\prime}}, \delta} \frac{d \mu^{p^{\prime}}}{d \nu} d \nu(x)\right]^{1 / p^{\prime}}
$$

This implies (2.31).

We also have

THEOREM 2.10. Under the same hypotheses, if $p \leq q$, then

$$
\begin{aligned}
\left\|M_{\sigma, \delta} f d \mu\right\|_{q, \omega} \leq & C\|f\|_{p, \nu} \sup _{|Q| \leq \delta^{n}}\left\|M_{\tau, \delta}(d \mu / d \nu)^{r^{\prime}} d \nu\right\|_{b / r^{\prime}, \omega, Q}^{1 / r^{\prime}} \\
& \times \sup _{|Q| \leq \delta^{n}}\left\|M_{\rho, \delta} \chi_{Q} d \nu\right\|_{a / r, \omega, Q}^{1 / r} \nu(Q)^{-1 / p} .
\end{aligned}
$$

ProOF. First we note that for any cube $Q$

$$
\chi_{Q} M_{\sigma, \delta} f d \mu \leq M_{\sigma, \delta} \chi_{Q+2 \delta} f d \mu .
$$

Let $\left\{I_{k}\right\}$ be a covering of $\mathbf{R}^{n}$ by disjoint cubes of side length $\delta$. Then by Corollary 2.6

$$
\begin{aligned}
\left\|M_{\sigma, \delta} f d \mu\right\|_{q, \omega} & =\left(\sum_{k}\left\|M_{\sigma, \delta} f d \mu\right\|_{q, \omega, I_{k}}^{q}\right)^{1 / q} \\
& \leq \sup _{k}\left\|M_{\tau, \delta} \frac{d \mu^{r^{\prime}}}{d \nu} d \nu\right\|_{b / r^{\prime}, \omega, I_{k}}^{1 / r^{\prime}}\left(\sum_{k}\left\|M_{\rho, \delta} f^{r} d \nu\right\|_{a / r, \omega, I_{k}}^{q / r}\right)^{1 / q}
\end{aligned}
$$


By (2.33) and Theorem 2.7, this last expression is bounded by

$$
\begin{aligned}
\left(\sum_{k}\left\|M_{\rho, \delta} \chi_{3 I_{k}} f^{r} d \nu\right\|_{a / r, \omega}^{q / r}\right)^{1 / q} \leq & \sup _{|Q| \leq \delta^{n}} \nu(Q)^{-1 / p}\left\|M_{\rho, \delta} \chi_{Q} d \nu\right\|_{a / r, \omega, Q}^{1 / r} \\
& \times\left(\sum_{k}\left\|\chi_{3 I_{k}} f^{r} d \nu\right\|_{p / r, \nu}^{q / r}\right)^{1 / q} .
\end{aligned}
$$

Since $p \leq q$, the last expression is bounded by

$$
\left(\sum_{k}\left\|\chi_{3 I_{k}} f^{r} d \nu\right\|_{p / r, \nu}^{p / r}\right)^{1 / p}=\left(3^{n} \sum_{k}\left\|\chi_{I_{k}} f^{r} d \nu\right\|_{p / r, \nu}^{p / r}\right)^{1 / p}=3^{n / p}\|f\|_{p, \nu} .
$$

If we combine these inequalities, we obtain (2.32).

PROPOSITION 2.11. If $\mu$ is absolutely continuous with respect to $\nu$ and

$$
\widetilde{G}_{r} d \mu(x)=\int_{|x-y|>r} G(x-y) d \mu(y),
$$

then

$$
\left\|\widetilde{G}_{r} f d \mu\right\|_{q, \omega} \leq\|f\|_{p, \nu}\left(\int\left(\int_{|x-y|>r} G(x-y)^{q} d \omega(x)\right)^{p^{\prime} / q} \frac{d \mu^{p^{\prime}}}{d \nu} d \nu\right)^{1 / p^{\prime}}
$$

ProOF. We have

$$
\begin{aligned}
\left\|\widetilde{G}_{r} f d \mu\right\|_{q, \omega} & =\left(\int\left(\int_{|x-y|>r} G(x-y) f(y) d \mu(y)\right)^{q} d \omega(x)\right)^{1 / q} \\
& \leq \int\left(\int_{|x-y|>r} G(x-y)^{q} d \omega(x)\right)^{1 / q} \frac{d \mu}{d \nu} d \nu(y) \\
& \leq\left(\int\left(\int_{|x-y|>r} G(x-y)^{q} d \omega(x)\right)^{p^{\prime} / q} \frac{d \mu^{p^{\prime}}}{d \nu} d \nu\right)^{1 / p^{\prime}}\|f\|_{p, \nu} .
\end{aligned}
$$

Now we give the proofs of the results of $\S 1$.

PROOF OF THEOREM 1.1. We note that (2.21) holds by Corollary 2.4. Then we apply Theorem 2.8 with $r=t$ and $\delta=\infty$.

PROOF OF THEOREM 1.2. We combine Corollary 2.4 with Theorem 2.9 taking $\delta=\infty$.

PROOF OF THEOREM 1.3. Here we combine Corollary 2.4 with Theorem 2.7 (again taking $\delta=\infty$ ).

PROOF OF THEOREM 1.4. Here we use Theorem 2.10.

PROOF OF COROLlARY 1.5. For $t \neq 1$, take $\rho=\sigma^{t} \tau^{1-t}$ in Theorem 1.4. Then

$$
M_{\tau}(d \mu / d \nu)^{t^{\prime}} d \nu=M_{\tau} d \tau=1 .
$$

If we take $b=\infty, a=q$, we obtain (1.15). For the case $t=1$, we use Theorem 1.3 . 
Next we show how we can weaken $(2.19)$ to

$$
\left\|\widetilde{G}_{R} d \mu_{Q}\right\|_{q, \omega}<\infty \text { for some } R \text { and all } Q .
$$

We have the following replacement for Corollary 2.4.

COROLlaRY 2.12. If $1 \leq q<\infty$ and (2.35) holds, then

$$
\|G f d \mu\|_{q, \omega} \leq q^{-q} 2^{q+1} C_{0}^{q}(q+1)^{q+1}\left\|M_{\sigma} f d \mu\right\|_{q, \omega} .
$$

ProOF. We follow the proofs of Corollaries 2.2-2.4. First assume $\left\|\widetilde{G}_{R} d \mu\right\|_{q, \omega}<$ $\infty$. Then by $(2.3)$

$$
\left\|G_{r} d \mu\right\|_{q, \omega} \leq \varepsilon\left\|G_{2 r} d \mu\right\|_{q, \omega}+2^{q+1} C_{0}^{q} \varepsilon^{-q}\left\|M_{\sigma} d \mu\right\|_{q, \omega} .
$$

Thus

$$
(1-\varepsilon)\left\|G_{r} d \mu\right\|_{q, \omega} \leq \varepsilon\left\|\widetilde{G}_{r} d \mu\right\|_{q, \omega}+2^{q+1} C_{0}^{q} \varepsilon^{-q}\left\|M_{\sigma} d \mu\right\|_{q, \omega}
$$

Since

$$
G_{r} d \mu \nearrow G d \mu, \quad \widetilde{G}_{r} d \mu \searrow 0
$$

we have in the limit

$$
(1-\varepsilon)\|G d \mu\|_{q, \omega} \leq \varepsilon^{-q} 2^{q+1} C_{0}^{q}\left\|M_{\sigma} d \mu\right\|_{q, \omega} .
$$

If we take $\varepsilon=q /(q+1)$, we obtain (2.36) for $f=1$. The general case is obtained as in the case of Corollaries 2.3 and 2.4 .

PROOF OF THEOREM 1.6. We replace Corollary 2.4 with Corollary 2.12 and follow the proofs as before.

Proof of THEOREM 1.7. We note that (1.18) implies (1.16) for $G(x)=G_{s}(x)$. In fact for $\beta<1$

$$
G_{s}(x)=O\left(e^{-\beta|x|}\right) \quad \text { as }|x| \rightarrow \infty .
$$

Thus the expression in (1.16) is bounded by a constant times

$$
\begin{gathered}
\left(\int\left(\int_{|x-y|>R ; y \in Q} e^{-\beta|x-y|} d y\right)^{q} d \omega(x)\right)^{1 / q} \\
\quad \leq \int_{Q}\left(\int_{|x-y|>R} e^{-q \beta|x-y|} d \omega(x)\right)^{1 / q} d y .
\end{gathered}
$$

This will be finite for $R$ sufficiently large if (1.18) holds. For $u \in H^{s, p}\left(\mathbf{R}^{n}, d \nu\right)$ let

$$
f=\bar{F}\left(1+|\xi|^{2}\right)^{s / 2} F u
$$

Then

$$
\|u\|_{s, p, \nu}=\|f\|_{p, \nu} \quad \text { and } \quad\|u\|_{q, \omega}=\left\|G_{s} f d x\right\|_{q, \omega} .
$$

We can now apply Corollary 1.5 and Theorem 1.6 to obtain (1.21). 


\section{BIBLIOGRAPHY}

1. E. T. Sawyer, A characterization of two-weight norm inequality for maximal operators, Studia Math. 75 (1982), 1-11.

2. B. Muckenhoupt and R. L. Wheeden, Two weight function norm inequalities for the HardyLittlewood maximal function and the Hilbert transform, ibid. 60 (1976), 279-294.

3. __ Weighted norm inequalities for fractional integrals, Trans. Amer. Math. Soc. 192 (1974), 261-275.

4. B. Muckenhoupt, Weighted norm inequalities for the Hardy maximal function, ibid. 156 (1972), 207-226.

5. S. Y. A. Chang, J. Wilson, and T. Wolff, Some weighted norm inequalities concerning the Schrödinger operators, Comment. Math. Helv. 60 (1985), 217-246.

6. S. Chanillo and R. L. Wheeden, $L^{p}$ estimates for fractional integrals and Sobolev inequalities with applications to Schrödinger operators, Comm. Partial Differential Equations 10 (1985), 1077-1116.

7. E. B. Fabes, C. Kenig and R. Serapioni, The local regularity of solutions of degenerate elliptic equations, ibid. 7 (1982), 77-116.

8. E. Stredulinsky, Weighted inequalities and degenerate elliptic partial differential equations, Lecture Notes in Math., vol. 1074, Springer, 1984.

9. S. Chanillo and R. L. Wheeden, Weighted Poincaré and Sobolev inequalities and estimates for weighted Peano maximal functions, Amer. J. Math. 107 (1985), 1191-1226.

10. V. G. Mazya, Imbedding theorems and their applications, Baku Symposium, "Nauka", Moscow, 1970, pp. 142-159.

11. D. R. Adams, On the existence of capacitary strong type estimates in $R^{n}$, Ark. Mat. 14 (1976), 125-140.

12. B. E. J. Dahlberg, Regularity properties of Riesz potentials, Indiana Univ. Math. J. 28 (1979), $257-268$.

13. M. Schechter, Hamiltonians for singular potentials, ibid. 22 (1972), 483-503.

14. D. R. Adams, Traces of potentials arising from translation invariant operators, Ann. Scuola Norm. Sup. Pisa 25 (1971), 203-217.

15. __ A trace inequality for generalized potentials, Studia Math. 48 (1973), 99-105.

16. J.-O. Stromberg and R. L. Wheeden, Fractional integrals on weighted $H^{p}$ and $L^{p}$ spaces, Trans. Amer. Math. Soc. 287 (1985), 293-321.

17. M. de Guzman, Differentiation of integrals in $R^{n}$, Lecture Notes in Math., vol. 481, SpringerVerlag, 1975.

18. R. Kerman and E. Sawyer, The trace inequality and eigenvalue estimates for Schrödinger operators, Ann. Inst. Fourier (to appear).

19. E. Sawyer, A weighted inequality and eigenvalue estimates for Schrödinger operators, Indiana J. Math. (to appear).

20. M. Schechter, On the invariance of the essential spectrum of an arbitrary operator II, Recerche Mat. 1 (1967), 3-26.

21. _ On the essential spectrum of an elliptic operator perturbed by a potential, J. Analyse Math. 22 (1969), 87-115.

22. M. S. Berger and M. Schechter, $L_{p}$ embedding and non-linear eigenvalue problems for unbounded domains, Bull. Amer. Math. Soc. 76 (1970), 1299-1302.

23. ___ Embedding theorems and quasilinear elliptic boundary value problems for unbounded domains, Trans. Amer. Math. Soc. 172 (1972), 261-278.

24. M. Schechter, Spectra of partial differential operators, North-Holland, Amsterdam, 1986.

25. V. G. Mazya and T. O. Shaposhnikova, Theory of multipliers in spaces of differentiable functions, Pitman, Marshfield, Mass., 1985.

26. E. T. Sawyer, A characterization of two weight norm inequalities for fractional and Poisson integrals (to appear).

Department of Mathematics, University of California, Irvine, California 92717 